\title{
FINAL STRUCTURED SUBSPACES
}

\author{
Amna M. A. Ahmed \\ Department of Mathematics, Al-Margib University, AlKhums, Libya \\ E-mail: danyaa2006@yahoo.com \\ Received: 22 August $2016 \quad$ Revised: 11 Nov 2017
}

Accepted: 18 Feb 2017

\begin{abstract}
Structured spaces or differential spaces are a generalization of the concept of smooth manifolds. Let $(M, \tau, C)$ be a structured space in the sense of Mostow and let $f:(M, \tau, C) \rightarrow N$ where $N$ is arbitrary, be a surjective function. There is a unique differential structure $D$ on $N$ determined by $f$ called the final, or identification differential structure, and the space $N$ then called the final structured space. In this paper, we will study structured subspaces of the final structured space $(N, D)$. The case when the subspace of the space $(N, D)$ is open is studied; and we prove that this subspace is also final. Some related concepts are defined and important properties are proved.
\end{abstract}

KEYWORDS Diffeomorphism, Differential Structure, Smooth map, Final Structured Space, Structured Subspace.

2010 Mathematics Subject Classification: 57R10, 58A40

\section{INTRODUCTION}

Structured spaces or differential spaces are a generalization of the concept of smooth manifolds (Heller \& Sasin, 1995b; Mostow, 1979). A structured space in the sense of Mostow is defined to be a topological space with a sheaf of continuous real-valued functions which are closed with respect to composition with smooth Euclidean functions (Heller \& Sasin, 1995; Mostow, 1979). Let $(M, \tau, C)$ be a structured space in the sense of Mostow and let $f:(M, \tau, C) \rightarrow N$ where $N$ is arbitrary, be a surjective function. There is a unique differential structure $D$ on $N$ determined by $f$ called the final, or identification, structured space (Bulati \& Ahmed, 2007).
In this paper, we will define and study final structured subspace of the final structured space $(N, D)$; where this differential structure will also be final. We shall define some related concepts and prove some results concerning them.

\section{PRELIMINARIES}

Differential structures in the sense of Mostow are defined as follows. Definition 2.1. (Heller \& Sasin, 1995b; Mostow, 1979) Let $M$ be a topological space with a topology $\tau$. A sheaf $C$ of real continuous functions on $M$ is said to be a differential structure (or a structural sheaf) on $M$ if it satisfies the following condition : for any nonempty set 
$U \in \tau$, any sections $f_{1}, \ldots, f_{n} \in C(U)$, where $n \in \mathbb{N}$, and any function $\omega$ : $\mathbb{R}^{n} \rightarrow \mathbb{R}$ of class $C^{\infty}$, the composition $\omega \circ\left(f_{1}, \ldots, f_{n}\right)$ belongs to $C(U)$. The ordered pair $(M, C)$, or the triple $(M, \tau, C), \quad$ is called a structured space.

Local functions are defined as following (Heller \& Sasin, 1995b).

Definition 2.2. Let $D$ be a presheaf of functions on a topological space $(M, \tau)$. For any nonempty open set $U \in \tau$, a function $f: U \rightarrow \mathrm{R}$ is a local $D$ function, if for any point $p \in U$ there exists a neighborhood $V$ of $p$ and a section $g \in D(V)$, such that $f \mid U \cap V=$ $g \mid U \cap V$. The set of all local $D$ - functions on $U$ will be denoted by $D_{M}(U)$.

A structured subspace of a structured space is defined as follows (Heller \& Sasin, 1995b; Mostow, 1979).

Definition 2.3. Let $(M, \tau, C)$ be any structured space and let $\left(A, \tau_{A}\right)$ be a topological subspace of $(M, \tau)$. Then $\left(A, \tau_{A}, C_{A}\right) \quad$ is called a structured subspace of $(M, \tau, C)$; where $C_{A}=$ $(C \mid A)_{A}$ and

$$
\begin{aligned}
(C \mid A)(V)= & \{f \mid V: f \in C(U), V \\
& =U \cap A \\
& \neq \varnothing, \text { for some } U \in \tau\} .
\end{aligned}
$$

Definition 2.4. (Heller \& Sasin, 1995b; Mostow, 1979) Let $(M, C)$ and $(N, D)$ be structured spaces. A continuous mapping $h: M \rightarrow N$ is said to be smooth provided $g \circ h \in C\left(h^{-1}(U)\right)$ for every section $g \in D(U)$.

Definition 2.5. (Gruszczak et al., 1988) Let $(M, C)$ and $(N, D)$ be structured spaces. A bijective mapping $h: M \rightarrow N$ is said to be a diffeomorphism provided both mappings $h: M \rightarrow N$ and $h^{-1}$ : $N \rightarrow M$ are smooth. Then $(M, C)$ and $(N, D)$ are said to be diffeomorphic.

Let $\left\{\left(M_{\alpha}, \tau_{\alpha}, C_{\alpha}\right)\right\}$ be a collection of structured spaces and $\left\{f_{\alpha}: M_{\alpha} \rightarrow N\right\}$, where $N$ is arbitrary, be a collection of functions. T. Bulati and A. M. A Ahmed defined final structured space as follows (Bulati \& Ahmed, 2007):

Definition 2.6. Let $\left\{\left(M_{\alpha}, C_{\alpha}\right)\right\}$ be a collection of structured spaces and $\left\{f_{\alpha}: M_{\alpha} \rightarrow N\right\}$ be a collection of functions. Let $\tau_{f}$ be the final topology on $\quad N$ with respect to $\left\{f_{\alpha}\right\}$. A differential structure $D$ on $\left(N, \tau_{f}\right)$ is said to be final with respect to the functions $\left\{f_{\alpha}\right\}$ if, for any structured space $(K, F)$ and function $h:(N, D) \rightarrow$ $(K, F)$, we have $h$ is smooth if and only if $h \circ f_{\alpha}:\left(M_{\alpha}, C_{\alpha}\right) \rightarrow$ 
$(K, F)$ is smooth for each $\alpha$. In this case, $\left(N, \tau_{f}, D\right)$, or $(N, D)$, is called the final structured space with respect to $\left\{f_{\alpha}\right\}$.

The following theorem shows that final differential structures always exist.

Theorem 2.7. (Bulati \& Ahmed, 2007) Let $\left\{\left(M_{\alpha}, C_{\alpha}\right)\right\}$ be a collection of structured spaces and $\left\{f_{\alpha}: M_{\alpha} \rightarrow N\right\}$ be a collection of functions. Then the final differential structure $D$ on $\left(N, \tau_{f}\right)$ with respect to $\left\{f_{\alpha}\right\}$ exists and is characterized by the following condition : if $U \in$ $\tau_{f}$, then $h \in D(U)$ if and only if $h \circ f_{\alpha} \in C_{\alpha}\left(f_{\alpha}^{-1}(U)\right)$ for each $\alpha$.

Definition 2.8. (Bulati \& Ahmed, 2007) Let $f:\left(M, \tau^{\prime}, C\right) \rightarrow(N, \tau, D)$ be any function. We say that $f$ is an identification mapping of structured spaces if $f$ is a surjection, $\tau=\tau_{f}$, and $D$ is the final differential structure on $N$ with respect to $f$. This differential structure on $\left(N, \tau_{f}\right)$ is also called the identification differential structure with respect to $f$, and we say $\left(N, \tau_{f}, D\right)$, or $(N, D)$, is the identification structured space with respect to $f$.

The identification differential structure $D$ on $\left(N, \tau_{f}\right)$ with respect to $f$ :
$(M, C) \rightarrow N$ is characterized as (Bulati \& Ahmed, 2007):

$$
\begin{gathered}
D(U)= \\
\left\{h: U \rightarrow \mathbb{R}: h \circ f \in C\left(f^{-1}(U)\right)\right\} .
\end{gathered}
$$

\section{FINAL STRUCTURED SUBSPACES}

Let $f:(M, C) \rightarrow(N, D)$ be an identification mapping of structured spaces, and let $A \subset N$. If $A$ is open in $N$, then the final topology on $A$ with respect to $f_{1}=f \mid f^{-1}(A), A$ : $f^{-1}(A) \rightarrow A$ is equal to $\tau_{A}$, the induced topology of $\tau$ (Dugundji, 1966). In this case, $\left(A, \tau_{A}\right)$ can receive two differential structures : (1) $D_{A}$, that as structured subspace of $(N, D)$; and (2) the final differential structure $F$ with respect to the surjection $f_{1}: f^{-1}(A) \rightarrow A$. Now, we will proof that the final differential structure $F$ with respect to $f_{1}$ and structured subspace of $(N, D)$ coincide. First, we have the following result.

Lemma 3.1. Let $f:(M, C) \rightarrow$ $(N, D)$ be an identification mapping of structured spaces and let $\left(A, D_{A}\right)$ be a structured subspace of $(N, D)$. Then the surjection $f_{1}=$ $f \mid f^{-1}(A), A:\left(f^{-1}(A), C_{f^{-1}(A)}\right) \rightarrow$ $\left(A, D_{A}\right)$ is smooth. 
Proof. Obviously, the map $f_{1}$ is continuous (see (Dugundji, 1966)). Let $h \in D_{A}(U)$. For each point $q \in f_{1}^{-1}(U)$, then $f_{1}(q) \in U$, so there exists a neighborhood $V$ of $f_{1}(q)$ and a section $g \in(D \mid A)(V)$ such that $h|W=g| W$, where $W=U \cap V$. Thus,

$$
(h \mid W) \circ f_{1}=(g \mid W) \circ f_{1} .
$$

Since $\quad(h \mid W) \circ f_{1}=\left(h \circ f_{1}\right) \mid f_{1}^{-1}(W)$ and $(g \mid W) \circ f_{1}=\left(g \circ f_{1}\right) \mid f_{1}^{-1}(W)$ we have

$$
\left(h \circ f_{1}\right)\left|f_{1}^{-1}(W)=\left(g \circ f_{1}\right)\right| f_{1}^{-1}(W),
$$

where $\quad f_{1}^{-1}(W)=f_{1}^{-1}(U \cap V)=$ $f_{1}^{-1}(U) \cap f_{1}^{-1}(V)$, and $g \circ f_{1}$ is a section of $C \mid f^{-1}(A)$ defined at $q$. So,

$$
h \circ f_{1} \in C_{f^{-1}(A)}\left(f_{1}^{-1}(U)\right) .
$$

Hence, the surjection $f_{1}:\left(f^{-1}(A), C_{f^{-1}(A)}\right) \rightarrow\left(A, D_{A}\right) \quad$ is smooth.

Theorem 3.2. Let $f:(M, \tau, C) \rightarrow$ $\left(N, \tau_{f}, D\right)$ be an identification mapping of structured spaces. Let $\left(A, D_{A}\right)$ be a structured subspace of $(N, D)$, where $A \in \tau_{f}$. Then the final differential structure $F$ on $A$ with respect to $f_{1}=f \mid f^{-1}(A), A: f^{-1}(A) \rightarrow A$ is equal to $D_{A}$.
Proof. By Lemma 3.1, $D_{A}(U) \subset F(U)$ for each nonempty set $U \in \tau_{A}$. Now, let $h \in F(U)$, then $h \circ f_{1} \in C_{f^{-1}(A)}\left(f_{1}^{-1}(U)\right)$. Since $f^{-1}(A)$ is open in $M, \quad C_{f^{-1}(A)}$ $=C \mid f^{-1}(A)$, so

$$
\begin{array}{r}
h \circ f=h \circ f_{1} \in C\left(f_{1}^{-1}(U)\right) \\
=C\left(f^{-1}(U)\right) .
\end{array}
$$

Since $D$ is the identification differential structure on $N$ with respect to $f$, we have $h \in D(U)$. Since $A$ is open in $N$, then $D_{A}=$ $D \mid A$, so $h \in D_{A}(U)$. Hence, $F(U) \subseteq D_{A}(U)$ for each nonempty set $U \in \tau_{A}$. Consequently, $\quad F=D_{A}$.

Definition 3.3. Let $f:(M, \tau, C) \rightarrow$ $\left(N, \tau_{f}, D\right)$ be an identification mapping of structured spaces, and let $A \in \tau_{f}$. Then $\left(A, \tau_{A}, D_{A}\right)$ is called the final structured subspace of $(N, D)$ with respect to $f$.

\section{QUOTIENT SUBSPACES}

Let $(M, \tau, C)$ be any structured space and let $\rho \subseteq M \times M$ be an equivalence relation in $(M, C)$. Let us consider the quotient space $(M / \rho, \tau / \rho)$ and the sheaf $C / \rho$ given by 


$$
\begin{aligned}
(C / \rho)(V)= & \left\{f: V \rightarrow \mathbb{R}: f \circ P_{\rho} \in\right. \\
& \left.C\left(P_{\rho}^{-1}(V)\right)\right\},
\end{aligned}
$$

for $V \in \tau / \rho$, where $P_{\rho}: M \rightarrow M_{\rho}$ is the canonical projection of the point $p$ onto its equivalent class (Heller \& Sasin, 1995a). The sheaf $C / \rho$ is the final differential structure on $(M / \rho, \tau / \rho)$ with respect to $P_{\rho}$, and $(M / \rho, \tau / \rho, C / \rho)$ is called the quotient structured space (Heller \& Sasin, 1995a).

Theorem 4.1. Let $\rho$ be an equivalence relation in $(M, C)$. If $A \subseteq M / \rho$ is open, then the structured spaces $\left(A,(C / \rho)_{A}\right)$ and $\left(P_{\rho}^{-1}(A) / \rho_{0}, C_{P_{\rho}^{-1}(A)} / \rho_{0}\right) \quad$ are diffeomorphic, where $\rho_{0}$ is the relation on $P_{\rho}^{-1}(A)$ induced by $\rho$.

Proof. By Theorem 3.2, since $A$ is open in $M / \rho, \quad(C / \rho)_{A}$ is the identification differential structure on $A$ with respect to the map:

$$
\begin{gathered}
f_{1}=P_{\rho} \mid P_{\rho}^{-1}(A), A:\left(P_{\rho}^{-1}(A), C_{P_{\rho}^{-1}(A)}\right) \rightarrow \\
\left(A,(C / \rho)_{A}\right) .
\end{gathered}
$$

Let $f_{2}=P_{\rho_{0}} ;$ that is, $f_{2}=$ $P_{\rho_{0}}:\left(P_{\rho}^{-1}(A), C_{P_{\rho}^{-1}(A)}\right) \rightarrow\left(P_{\rho}^{-1}(A) /\right.$ $\left.\rho_{0}, C_{P_{\rho}^{-1}(A)} / \rho_{0}\right)$ is the projection of the point $p$ onto its equivalent class $[p]_{\rho_{0}}$.

Obviously, both $f_{1}, f_{2}$ are continuous (see (Dugundji, 1966)). Notice that $f_{1} f_{2}^{-1}$ is single-valued [i.e., $f_{1}$ is constant on each $\left.f_{2}^{-1}(s), s \in P_{\rho}^{-1}(A) / \rho_{0}\right]$, so we have

$$
f_{1}(x)=f_{1} f_{2}^{-1} f_{2}(x)=\left(f_{1} f_{2}^{-1}\right) \circ f_{2}(x),
$$

for all $x \in P_{\rho}^{-1}(A)$; that is, $f_{1}=$ $\left(f_{1} f_{2}^{-1}\right) \circ f_{2}$. Because $f_{1}$ is smooth, we have $f_{1} f_{2}^{-1}$ is smooth since $f_{2}$ is the identification map of structured spaces (from Definition 2.6).

Similarly, $f_{2} f_{1}^{-1}:\left(A,(C / \rho)_{A}\right) \rightarrow$ $\left(P_{\rho}^{-1}(A) / \rho_{0}, C_{P_{\rho}^{-1}(A)} / \rho_{0}\right)$ is smooth. Since the smooth mappings $f_{1} f_{2}^{-1}$ and $f_{2} f_{1}^{-1}$ are inverses of one another, $\left(A,(C / \rho)_{A}\right) \quad$ and $\left(P_{\rho}^{-1}(A) /\right.$ $\left.\rho_{0}, C_{P_{\rho}^{-1}(A)} / \rho_{0}\right)$ are diffeomorphic.

Example 4.2. Let $(M, \tau, C)$ be any structured space. For $A \subseteq M$, let $E_{A}$ be the equivalence relation defined by

$$
E_{A}=(A \times A) \cup\{(x, x): x \in M\} .
$$

The quotient space $M / E_{A}$ is the space $M$ with $A$ identified to a point $E_{A} a$, for $a \in A$ (see (Dugundji, 1966)). If $A$ is closed, then $B=M-A$ is open in $M$. Hence, the set $B_{1}=$ $M / E_{A^{-}} E_{A} a$ is open in $M / E_{A}$, and the mapping $P_{E_{A}} \mid B, B_{1}$ is bijective. By Theorem 3.2, $\left(C / E_{A}\right)_{B_{1}}$ is the final differential structure on $\left(B_{1}, \tau_{B_{1}}\right)$ with respect to $P_{E_{A}} \mid B, B_{1}$. 


\section{CONCLUSION}

The study has shown some cases in which structured subspaces of final structured spaces are also final.

Interesting future studies and more properties can be investigated. Final mapping of structured spaces might be studied for special maps or special topological spaces. Closed subspaces might also be studied to show in which cases these subspaces are also final.

\section{REFERENCES}

Brown, R. (1968). Elements of Modern Topology, New York, USA: McGraw - Hill Book Company.

Bulati, T. \& Ahmed, A. (2007). Final structured spaces. Journal of Basic and Applied Sciences 10(3): $22-28$.
Dugundji, J. (1966). Topology , Boston: Allyn and Bacon, Inc.

Gruszczak, J., Heller, M. \& Multarzynski, P. (1988). A generalization of manifolds as space - time models. Journal of Mathematical Physics 29: $2576-2580$.

Heller, M. \& Sasin, W. (1995a). Anatomy of the elementary quasi- regular singularity. Acta Cosmologica Fasciculus 21: 47-60.

Heller, M. \& Sasin, W. (1995b). Structured spaces and their application to relativistic physics. Journal of Mathematical Physics 36: 3644-3662.

Mostow, M. (1979). The differentiable space structures of Milnor classifying spaces, simplicial complexes, and geometric realizations. J. Differential Geometry 14: 255-293. 